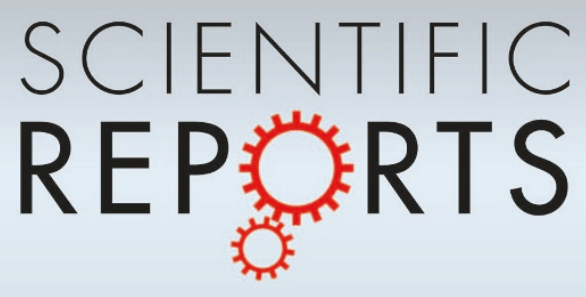

OPEN

SUBJECT AREAS:

ELECTRONIC PROPERTIES

AND MATERIALS

SUPERCONDUCTING PROPERTIES

AND MATERIALS

STRUCTURE OF SOLIDS AND

LIQUIDS

Received

22 May 2014

Accepted

15 July 2014

Published

30 July 2014

Correspondence and requests for materials should be addressed to

Z.R. (zamra51@ifm.

liu.se)

\section{Novel superconducting skutterudite-type phosphorus nitride at high pressure from first-principles calculations}

\author{
Zamaan Raza ${ }^{1,2}$, Ion Errea ${ }^{1,3,4}$, Artem R. Oganov ${ }^{5,6,7,8}$ \& A. Marco Saitta ${ }^{1,2}$
}

\begin{abstract}
'Sorbonne Universités, UPMC Univ. Paris 06, UMR 7590, Institut de Minéralogie, de Physique des Matériaux et de Cosmochimie (IMPMC), F-75005 Paris, France, ${ }^{2}$ CNRS, UMR 7590, Institut de Minéralogie, de Physique des Matériaux et de Cosmochimie (IMPMC), F-75005 Paris, France, ${ }^{3}$ Donostia International Physics Center (DIPC), Manuel de Lardizabal pasealekua 4, 20018 Donostia-San Sebastián, Basque Country, Spain, ${ }^{4}$ IKERBASQUE, Basque Foundation for Science, 48011 , Bilbao, Spain, ${ }^{5}$ Department of Geosciences, State University of New York, Stony Brook, NY 1 1794-2100, USA, ${ }^{6}$ Center for Materials Design, Institute for Advanced Computational Science, State University of New York, Stony Brook, NY 1 1794-201 1, USA, ${ }^{7}$ Moscow Institute of Physics and Technology, 9 Institutskiy Lane, Dolgoprudny City, Moscow Region, 141700, Russian Federation, ${ }^{8}$ Northwestern Polytechnical University, Xi'an, 710072, China.
\end{abstract}

State of the art variable composition structure prediction based on density functional theory demonstrates that two new stoichiometries of $\mathrm{PN}, \mathrm{PN}_{3}$ and $\mathrm{PN}_{2}$, become viable at high pressure. $\mathrm{PN}_{3}$ has a skutterudite-like Immm structure and is metastable with positive phonon frequencies at pressures between 10 and $100 \mathrm{GPa} . \mathrm{PN}_{3}$ is metallic and is the first reported nitrogen-based skutterudite. Its metallicity arises from nitrogen $\mathrm{p}$-states which delocalise across $\mathrm{N}_{4}$ rings characteristic of skutterudites, and it becomes a good electron-phonon superconductor at $10 \mathrm{GPa}$, with a $T_{c}$ of around $18 \mathrm{~K}$. The superconductivity arises from strongly enhanced electron-phonon coupling at lower pressures, originating primarily from soft collective $\mathrm{P}-\mathrm{N}$ phonon modes. The $\mathrm{PN}_{2}$ phase is an insulator with $P 2 / \mathrm{m}$ symmetry and is stable at pressures in excess of $200 \mathrm{GPa}$.

igh pressure synthesis offers a route to new materials with promising technological applications such as superconductors ${ }^{1-4}$ as well as to compounds with unexpected stoichiometries ${ }^{5}$. New theoretical structure prediction techniques $^{6-9}$ have been used to identify novel superconductors by applying pressure to stoichiometries that form phases without the properties of superconductivity, metallicity or even stability at atmospheric pressure ${ }^{10-15}$. In spite of their great potential, nitride-based materials remain relatively unexplored due to the high stability of nitrogen molecules at ambient pressure; the strong $\mathrm{N} \equiv \mathrm{N}$ triple bond results in a high kinetic barrier to polymerisation, thus extreme temperatures and pressures are required to synthesise many nitrides. However, at high pressures, the physical and chemical properties of nitrogen change substantially, allowing the formation of new polymeric crystalline phase ${ }^{16}$ and the possibility of forming new nitrogen-based superconducting crystals.

Nitride-based superconductors were among the first discovered, and include examples with remarkably high superconducting critical temperatures $\left(T_{c}\right)$, such as $\mathrm{NbN}(14.7 \mathrm{~K})^{17}$ and $\beta$ - $\mathrm{HfNCl}(25.5 \mathrm{~K})^{18}$. However, in both instances, the superconductivity does not originate from the nitrogen atoms ${ }^{19}$, and the $\mathrm{XNCl}$ family of layered compounds have been proposed to be unconventional superconductors ${ }^{20}$.

Another important example of superconductivity in pnictogen-based compounds involves the skutterudites, though, as far as we know, no nitrogen-based skutterudite has been found yet. They have the empirical formula $\mathrm{TX}_{3}$, where $\mathrm{T}$ is typically a transition metal and $\mathrm{X}$ is a pnictogen. They form a low density body-centred cubic $\operatorname{Im} \overline{3}$ lattice consisting of dodecahedral cages (Fig. 1). This cage can be filled with a guest atom to form $\mathrm{MT}_{4} \mathrm{X}_{12}$, potentially changing the properties of the material, making it semiconducting ${ }^{21}$, paramagnetic ${ }^{22}$,superconducting ${ }^{23}$ or even thermoelectric ${ }^{24}$. They are also characterised by square $\mathrm{X}_{4}^{4-}$ groups connecting the cages, which are thought to influence electrical conductivity and superconductivity ${ }^{25}$. Filled skutterudite superconducting materials have been synthesised under pressure ${ }^{26}$, and found to have high $T_{c}$ values, for example $\mathrm{LaRu}_{4} \mathrm{As}_{12}$ with $T_{c}=10.3 \mathrm{~K}^{26}$ and $\mathrm{La}_{0.6} \mathrm{Rh}_{4} \mathrm{P}_{12}$ with $T_{c}=17 \mathrm{~K}$, the highest known among metal phosphides ${ }^{27}$. It has been shown that although the guest atom in filled skutterudites stabilises the lattice, the superconductivity is an intrinsic property of the $\mathrm{T}_{4} \mathrm{X}_{12}$ framework ${ }^{28,29}$. 

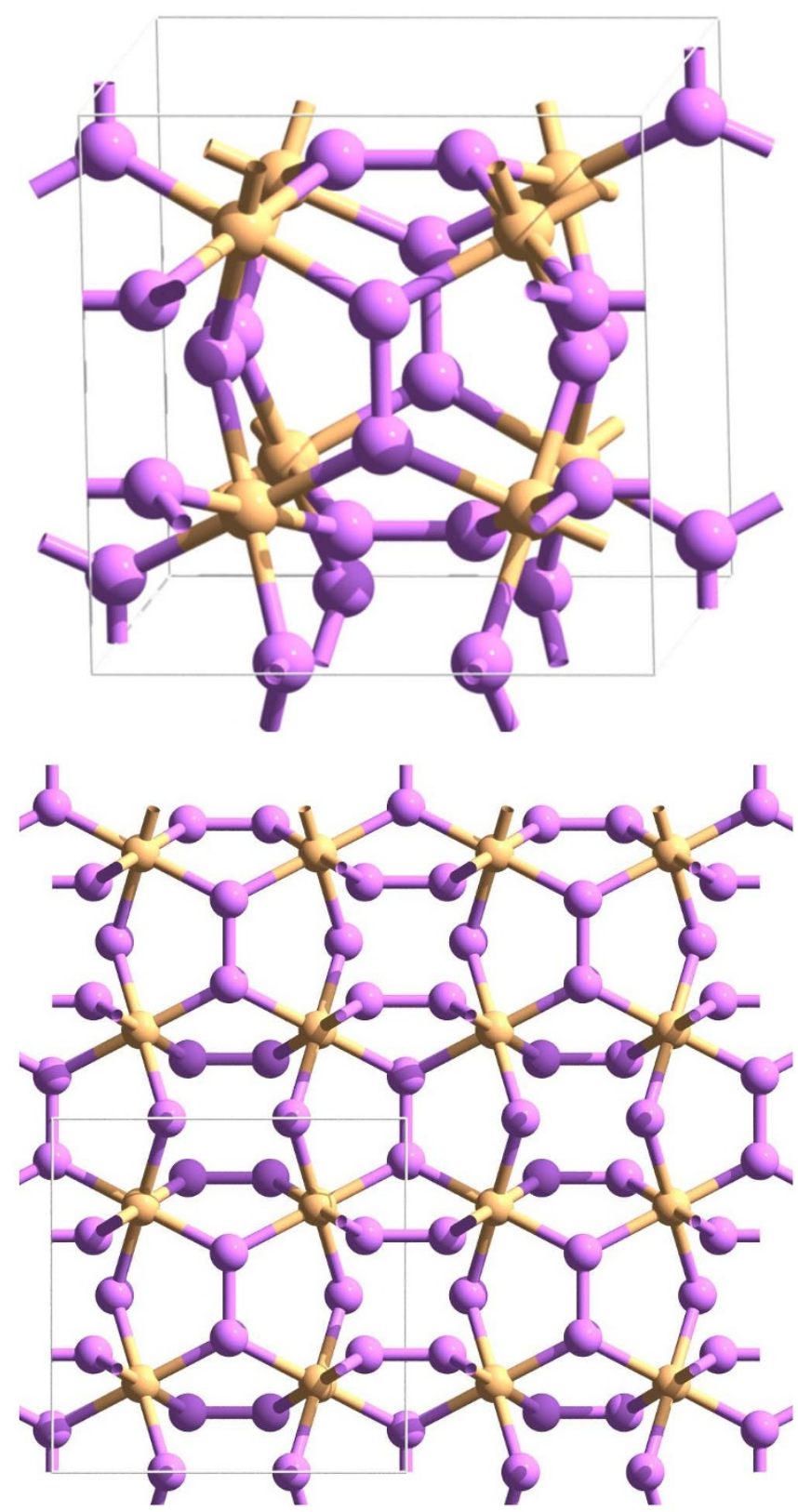

Figure 1 The skutterudite-like Immm structure of $\mathrm{PN}_{3}$ in its bodycentred orthorhombic conventional cell. The skutterudite structure has $\operatorname{Im} \overline{3}$ symmetry, but the $\mathrm{PN}_{3}$ phase is characterised by an orthorhombic distortion. Phosphorus atoms are six-coordinated in both cases. Phosphorus atoms are yellow and nitrogen atoms purple.

In this article we perform a systematic search for thermodynamically stable phosphorus nitrides under pressure using evolutionary structure prediction and density functional theory (DFT). Beyond the only known stable phosphorus nitride, $\mathrm{P}_{3} \mathrm{~N}_{5}$, which forms a variety of pressure-dependent phases $^{30-33}$, we find that a novel phosphorus nitride skutterudite $\left(\mathrm{PN}_{3}\right)$ containing six-coordinated phosphorus can be formed at undemanding pressures $(\sim 10 \mathrm{GPa}) . \mathrm{PN}_{3}$ is the first reported nitrogen-based skutterudite. It is metallic, metastable and dynamically stable between 10 and $100 \mathrm{GPa}$. Although it is unstable at ambient pressure, it may be possible to stabilise it using offstoichiometry doping with a guest species at the centre of the cage, as with other skutterudite superconductors. $\mathrm{PN}_{3}$ is a good superconductor with a $T_{c}$ of around $18 \mathrm{~K}$ at $10 \mathrm{GPa}$, higher than for any known skutterudite. We also report the discovery of a new insulating phase with $\mathrm{PN}_{2}$ stoichiometry, which is stable above $200 \mathrm{GPa}$.

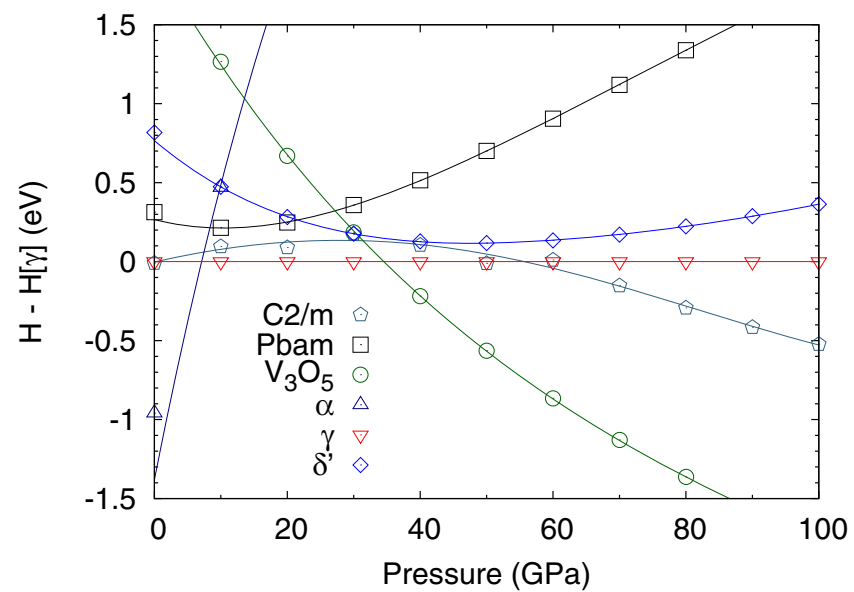

Figure $2 \mid$ Enthalpy per formula unit as a function of pressure for the best phases with the $\mathrm{P}_{3} \mathrm{~N}_{5}$ stoichiometry.

\section{Results}

High pressure phosphorus nitride structures. In the first instance, fixed composition searches were performed using one and two formula units of $\mathrm{P}_{3} \mathrm{~N}_{5}$ at pressures of $25 \mathrm{GPa}, 50 \mathrm{GPa}, 100 \mathrm{GPa}$ and $200 \mathrm{GPa}$, yielding the $\operatorname{Imm} 2 \gamma$-phase at lower pressures, and the previously predicted $C 2 / c$ oxyvanite phase at high pressures, in agreement with the findings of Dong et al. ${ }^{34}$ The recovery of an experimentally verified phase in addition to a structure predicted through chemical intuition is a reassuring vindication of the USPEX code. According to the results presented in Fig. 2, the $\alpha$ phase, which is stable at ambient pressure, undergoes a transition to the $\gamma$ phase at around $7 \mathrm{GPa}$, which transforms to the oxyvanite phase at $34 \mathrm{GPa}$. The previously predicted $\delta^{\prime}$ phase $^{35}$ is at least $0.2 \mathrm{eV}$ higher in enthalpy than $\gamma$.

Variable composition searches were performed at $100 \mathrm{GPa}$ in order to identify potential stable stoichiometries; these were followed by fixed composition searches for the best stoichiometries at pressures of $25 \mathrm{GPa}, 50 \mathrm{GPa}, 100 \mathrm{GPa}$ and $200 \mathrm{GPa}$ using $\mathrm{PN}_{3}, \mathrm{PN}_{2}$, $\mathrm{P}_{3} \mathrm{~N}_{4}, \mathrm{P}_{3} \mathrm{~N}_{5}$ and $\mathrm{PN}$. The resulting convex hull is shown in the

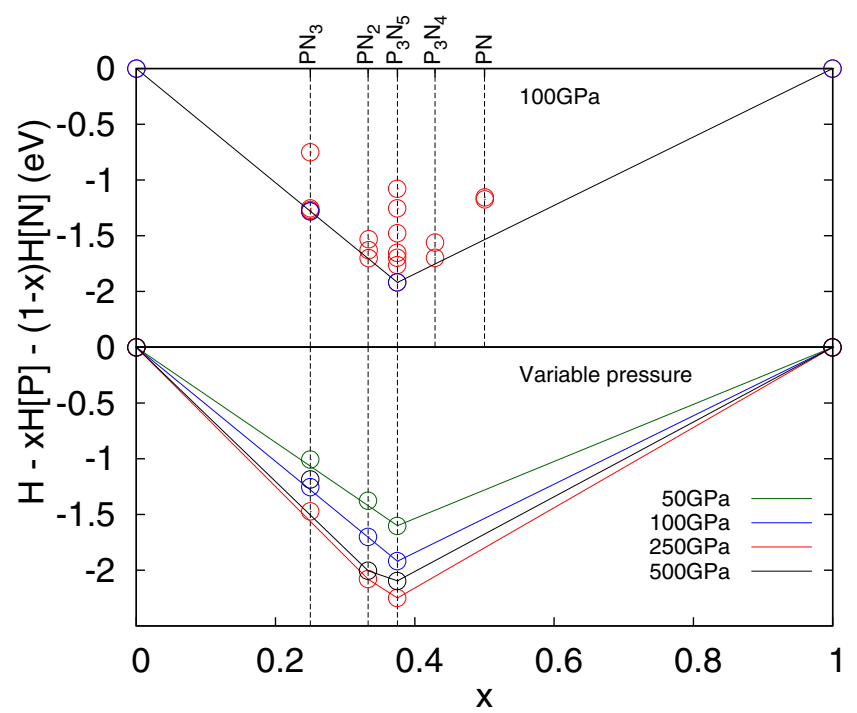

Figure $3 \mid$ Convex hull for the $\mathrm{P}_{\mathrm{x}} \mathrm{N}_{1-\mathrm{x}}$ system at $100 \mathrm{GPa}$ (top) and at different pressures (bottom). $\mathrm{H}$ is the enthalpy per atom of the generated structure, $\mathrm{H}[\mathrm{P}]$ the enthalpy per atom of the stable phosphorus phase (simple hexagonal phosphorus at $100 \mathrm{GPa}$ ) and $\mathrm{H}[\mathrm{N}]$ is the enthalpy per atom of the stable nitrogen phase (cubic gauche nitrogen at $100 \mathrm{GPa}$ ). The lower panel only includes the Immm- $\mathrm{PN}_{3}, P 2 / m-\mathrm{PN}_{2}$ and oxyvanite- $\mathrm{P}_{3} \mathrm{~N}_{5}$ structures. 


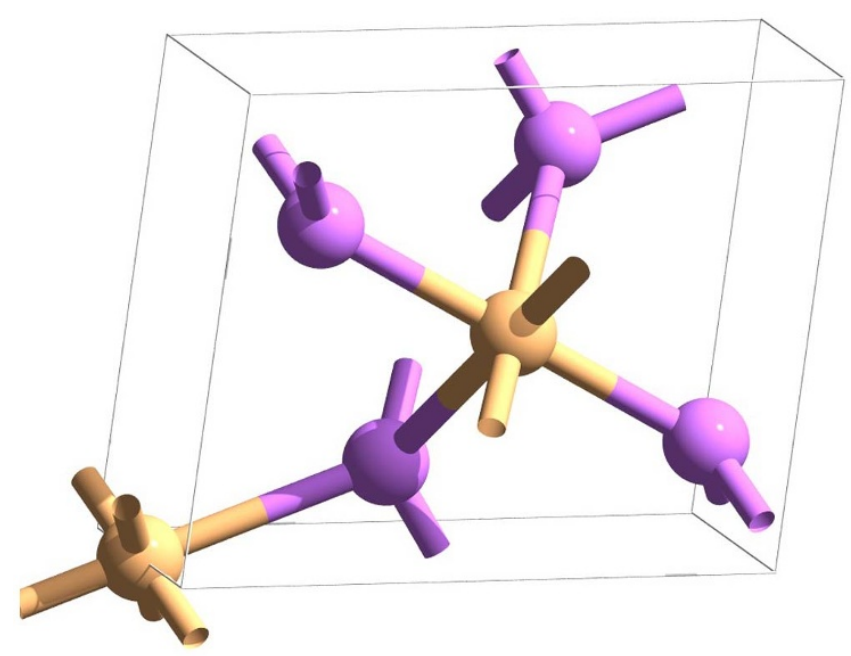

Figure $4 \mid$ The $P 2 / m$ structure of $\mathrm{PN}_{2}$, which becomes stable at pressures of above $200 \mathrm{GPa}$.

upper panel of Fig. 3, illustrating the most stable compositions: $\mathrm{PN}_{3}$, $\mathrm{PN}_{2}$ and $\mathrm{P}_{3} \mathrm{~N}_{5}$. The enthalpies of the most interesting structures are plotted at different pressures in the lower panel. A new insulating P2/ $m$ phase of $\mathrm{PN}_{2}$ (structure in Fig. 4 and electronic density of states in Fig. 5) becomes stable with respect to $\mathrm{N}$ and $\mathrm{P}_{3} \mathrm{~N}_{5}$ at pressures in excess of $200 \mathrm{GPa}$, while the best $\mathrm{PN}_{3}$ structure $(\mathrm{Immm})$ is very close to the hull below $100 \mathrm{GPa}$. It is noteworthy that both new structures consist of octahedral $\mathrm{PN}_{6}$ units, containing octahedral six-coordinated phosphorus atoms; this was unprecedented until a pressure induced transition to six-coordinated phosphorus was observed in $\mathrm{AlPO}_{4}$ as a densification mechanism ${ }^{36}$. The structural parameters for these two new structures are listed in Table I.

The Immm- $\mathrm{PN}_{3}$ skutterudite. The skutterudite-like $\mathrm{PN}_{3}$ is formed from dodecahedral cages (Fig. reffig:Immm-structure) consisting of six-coordinated phosphorus atoms at the vertices of a cuboid, with the faces occupied by $\mathrm{N}_{2}$ pairs, forming pentagonal rings. In contrast with all other known and predicted PN structure, the nitrogen atoms are all three-coordinated, compared with the $\gamma$, oxyvanite and new $\mathrm{P} 2 / \mathrm{m}$ phases, which contain both three- and four-coordinated nitrogens. Surprisingly, the N-N bonds are extremely short; at $100 \mathrm{GPa}$, they are $1.28 \AA$, increasing to $1.30 \AA$ at $10 \mathrm{GPa}$. No other structures were found with such a short bond length. As the pressure decreases, the orthorhombic distortion in the Immm cell becomes more pronounced in the $b$ direction, causing the structure to break down; it is likely that this is thermodynamically favourable since the $\mathrm{N}_{2}$ units are very close in geometry to their configuration in the gas phase. However, the structure is dynamically stable with well-defined positive phonons at pressures as low as $10 \mathrm{GPa}$. Thus the possibility remains that the skutterudite structure may be synthesised at moderate pressures.

$\mathrm{MX}_{3}$ skutterudites are also characterised by six square $\mathrm{X}_{4}$ rings in the conventional 32-atom cell. In $\mathrm{PN}_{3}$, these rings are rectangular rather than square as a result of the orthorhombic distortion, but can be discerned as pairs of $\mathrm{N}_{2}$ units separated by $1.9 \AA$ at $100 \mathrm{GPa}$, which could be either a weak bond or a non-bonding contact. A Bader analysis reveals that the nitrogens have little ionic character, with a charge of 0.065 to $0.075 e$. These $\mathrm{X}_{4}$ rings have been shown to give rise to conduction and superconductivity in other skutterudites, namely $\mathrm{CoP}_{3}$ and $\mathrm{NiP}_{3}{ }^{37}$ and $\mathrm{LaFe}_{4} \mathrm{P}_{12}{ }^{25}$.

$\mathrm{PN}_{3}$ is metallic throughout its stable pressure range. There is a distinct set of three bands (Fig. 6) dispersing from approximately $1 \mathrm{eV}$ below $\epsilon_{F}$ to $2 \mathrm{eV}$ above $\epsilon_{F}$ responsible for the conductivity. The partial density of states (Fig. 6) demonstrates that the dominant

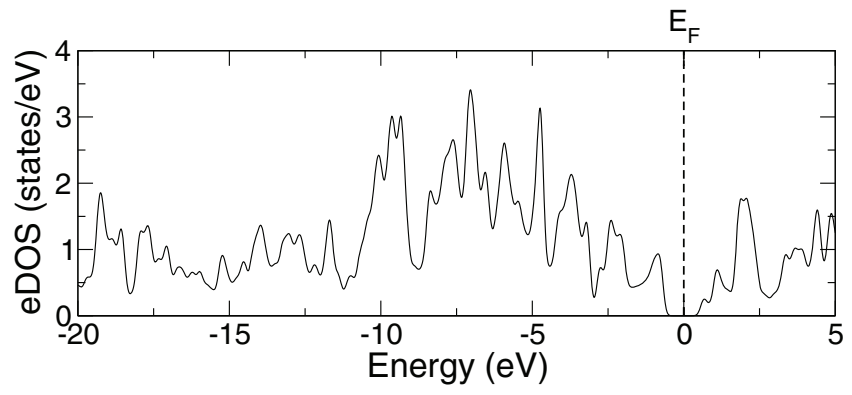

Figure 5 | Electronic density of states of the $P 2 / m$ phase at $200 \mathrm{GPa}$.

contribution to the conducting bands arises from nitrogen $2 \mathrm{p}$ atomic orbitals. It is interesting to note that in electronic structure calculations of other metallic skutterudites, namely $\mathrm{CoP}_{3}$ and $\mathrm{NiP}_{3}$, the highest occupied bands consist of phosphorus centred p-orbitals ${ }^{37}$, which occupy analogous positions to nitrogen in $\mathrm{PN}_{3}$, phosphorus being isoelectronic to nitrogen.

The three bands around the Fermi level were projected onto maximally localised Wannier functions (MLWF), as illustrated in Fig. 7. The Wannier projections have p-orbital-like symmetry, but are delocalised across $\mathrm{N}_{2}$ pairs which form adjacent skutterudite $\mathrm{N}_{4}$ rings, accounting for the conductivity.

Phonon spectra and electron-phonon coupling in the Immm- $\mathrm{PN}_{3}$ skutterudite. The phonon spectra of $\mathrm{PN}_{3}$ (Fig. 8) are characterised by two groups of modes; firstly dispersive low-energy modes that involve collective vibrations of $\mathrm{P}$ and $\mathrm{N}$ atoms. These modes are separated from the second group, high-energy Einstein-like modes, primarily corresponding to vibrations of the short $\mathrm{N}-\mathrm{N}$ bonds, by a distinct gap. As the pressure is lowered, the acoustic modes and some low-energy optical modes related to distortions of the whole dodecahedral cage are softened. This gives rise to a structural instability at ambient pressure as the lowest energy mode at the $\mathrm{X}$ and $\mathrm{R}$ point acquire an imaginary frequency. The instability is related to the decomposition of $\mathrm{PN}_{3}$ and the formation of $\mathrm{N}_{2}$ pairs. Structurally, this is manifested as the formation of PN "sheets" enclosing $\mathrm{N}_{2}$ molecules, and is reflected by significant changes in the phonon dispersion. However, at $10 \mathrm{GPa}$ the phonon spectrum does not display any imaginary frequencies and the crystal structure is dynamically stable.

The electron-phonon coupling constant $\lambda$, calculated from equation (3), scales linearly with the density of states at the Fermi level and is inversely proportional to the square of the phonon frequencies. Its calculated values are presented in Table II and the Eliashberg functions are plotted in Fig. 9. At all pressures studied, the eDOS at $\epsilon_{F}$ remains high due to the presence of weakly dispersive bands in the vicinity of the Fermi level (Fig. 6). This ensures a large electronic contribution to the Eliashberg functions coming from the double electronic Dirac delta in equation (1). Thus, the evolution of $\lambda$ under pressure is mostly determined by the evolution of the phonon spectra.

At $10 \mathrm{GPa}$ the electron-phonon coupling constant reaches a remarkable value of 1.05 . The low-energy modes $\left(<400 \mathrm{~cm}^{-1}\right)$ contribute more strongly to $\lambda$ due to their low frequencies. This is evidenced by the strong contribution of the low-energy part of the Eliashberg function to $\lambda$, demonstrated in Fig. 9. The contribution to $\lambda$ of the lowest energy acoustic mode at the $\mathrm{R}$ special point is particularly large. At $20 \mathrm{GPa}$, the electron-phonon coupling decreases as low-frequency modes gain energy. This causes the strong peaks in $\alpha^{2} F(\omega)$ at low energy to shift to higher frequencies and thus reduces their contribution to $\lambda$. At higher pressures, the energy of the lowest energy acoustic mode at R is strongly increased and, consequently, its contribution to $\lambda$ is strongly suppressed, indu- 
Table I | Structural parameters for the two new PN phases

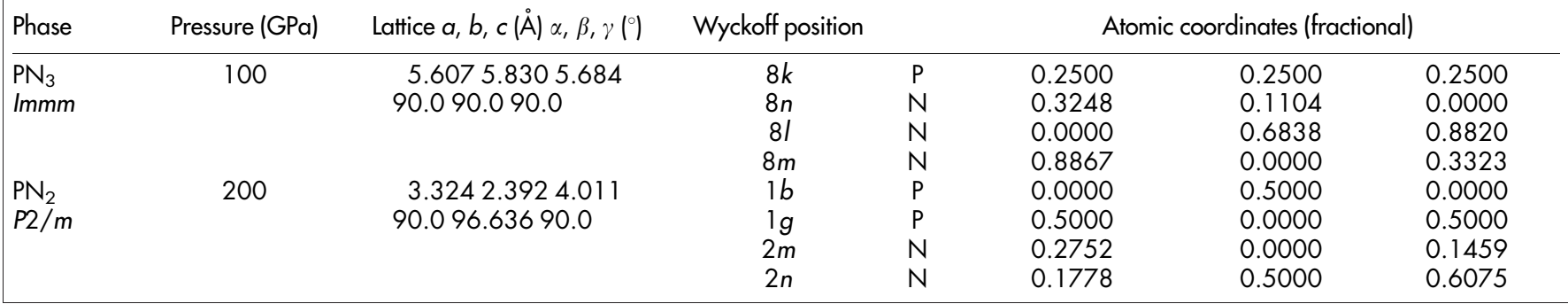

cing a considerable reduction of the electron-phonon coupling constant.

Using the computed values of the electron-phonon coupling constant, we estimated the superconducting $T_{c}$ using the standard values of $\mu^{*}=0.10$ and $\mu^{*}=0.13$ for the Coulomb pseudopotential in equation (2). These values are summarised in Table II. $T_{c}$ reaches $18.6 \mathrm{~K}$ for $\mu^{*}=0.10$ at $10 \mathrm{GPa}$, a high value for an electron-phonon coupling superconductor. Using $\mu^{*}=0.13$, a slightly lower but similar $16.1 \mathrm{~K}$ value is obtained, showing that for large values of $\lambda T_{c}$ is weakly dependent on $\mu^{*}$. The evolution of $T_{c}$ is consistent with the pressure dependence of $\lambda$ and both are suppressed at high pressures.

Inclusion of a guest atom in the skutterudite lattice. Filled skutterudites are of particular interest for their potential thermoelectric properties. If the void in a skutterudite cage is significantly larger than the guest atom, then the vibrational modes of the guest atom are expected to be strongly anharmonic, resulting in "rattling modes"24. These modes tend to reduce thermal conductivity by scattering the phonons responsible for heat transport ${ }^{38}$ and, consequently, enhance the figure of merit of thermoelectric materials ${ }^{39}$. We now turn to the question of whether the skutterudite remains stable when it hosts such a guest atom.
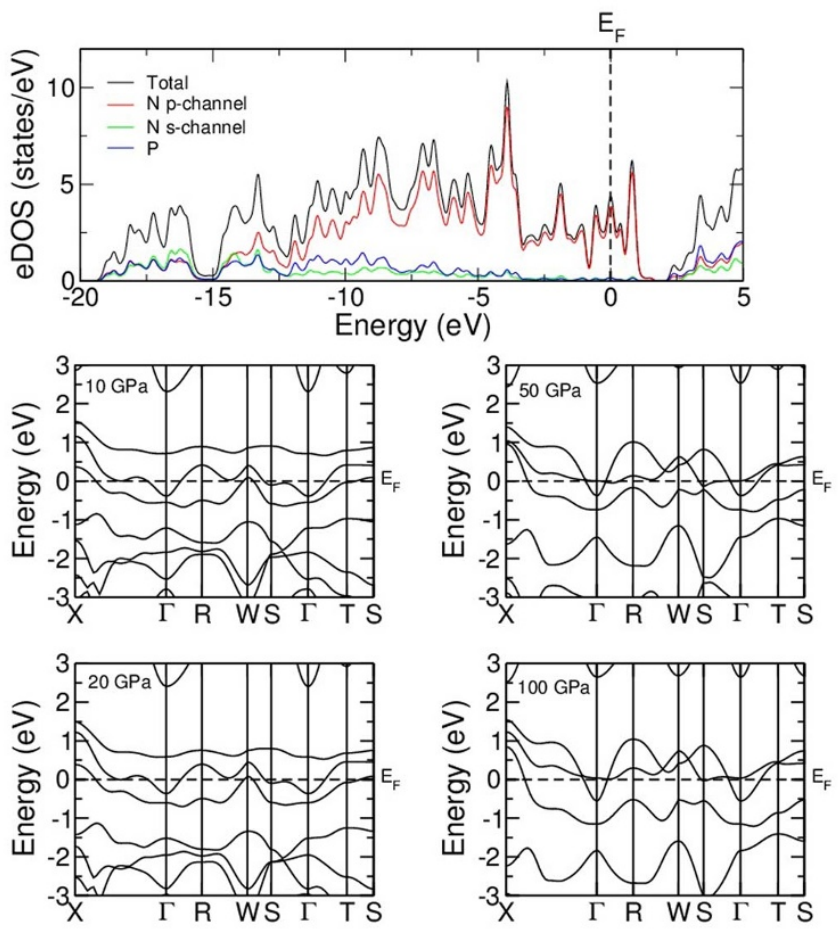

Figure 6 Electronic structure of the $I m m m P_{3}$ phase. The electronic density of states (top panel) is decomposed into contributions from $\mathrm{P}$ atoms, $\mathrm{N} 2 \mathrm{~s}$ electons and $\mathrm{N} 2 \mathrm{p}$ electrons. The band structure near the Fermi level is displayed at $10 \mathrm{GPa}, 20 \mathrm{GPa}, 50 \mathrm{GPa}$ and $100 \mathrm{GPa}$.
It should be noted that the cage in $\mathrm{PN}_{3}$ is considerably smaller than for other skutterudites, which are large enough to hold atoms such as barium, which has a covalent radius of $2.53 \AA$. $\mathrm{PN}_{3}$ has a bodycentred orthorhombic lattice at lower pressures, in contrast with most skutterudites which are body-centred cubic; the distance between the body centre of the cage and the closest point on the surface of the cage (specifically, the midpoint of a $\mathrm{N}_{2}$ bond) is 1.81 $\AA$, compared with $2.79 \AA$ for $\mathrm{CoAs}_{3}$. This greatly limits the choice of guest atom to smaller species such as hydrogen, lithium and beryllium.

A beryllium atom proved to be too large, resulting in imaginary frequencies throughout the Brillouin zone. A lone hydrogen atom was too thermodynamically unstable to occupy the body centre of the lattice, drifting towards a phosphorus atom during geometry optimisation and breaking the Immm symmetry. The phonon dispersion for $\mathrm{PN}_{3}$ with a lithium guest has a soft mode at the $\mathrm{X}$ special point, and is therefore not stable; this was the case through the pressure range $10-100 \mathrm{GPa}$. In conclusion, it seems there is no stable $\mathrm{XP}_{4} \mathrm{~N}_{12}$ filled skutterudite.

The inclusion of a guest atom and a reduction of the external pressure have a similar effect on the atomic structure: the guest atom forces the cage to expand, just as the structure loses its dynamical stability as the lattice parameter increases at pressures below $10 \mathrm{GPa}$. It may be possible to stabilise the structure by doping the system with, for example, one lithium atom per eight conventional cells (i.e. a $2 \times$ $2 \times 2$ supercell), although this would necessitate phonon calculations that would be prohibitively expensive.

\section{Discussion}

In conclusion, we used density functional theory coupled with evolutionary structure prediction to explore the variable composition configuration space of phosphorus nitride. We identified a new stable stoichiometry, insulating $\mathrm{PN}_{2}$ with $P 2 / m$ symmetry, at pressures in excess of $200 \mathrm{GPa}$, and a fascinating new metastable structure, $\mathrm{PN}_{3}$, which is dynamically stable between approximately 10 and $100 \mathrm{GPa}$.

The $\mathrm{PN}_{3}$ structure has Immm symmetry, belongs to the $\operatorname{Im} \overline{3}$ skutterudite class of compounds, and is the first to contain nitrogen as

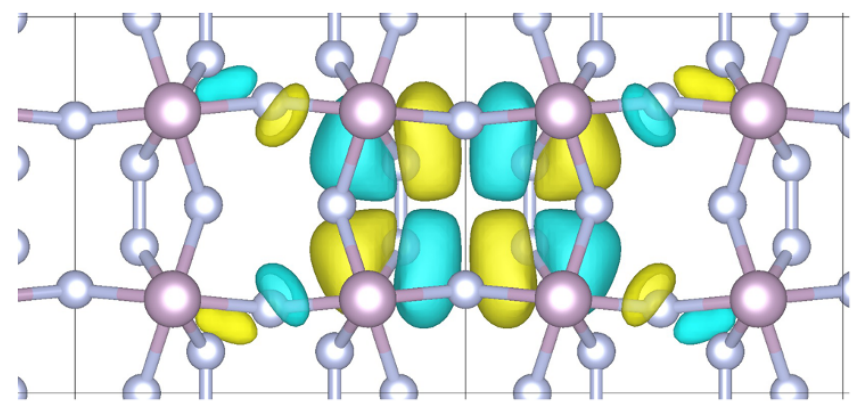

Figure $7 \mid$ A MLWF projection of the states from $1 \mathrm{eV}$ below to $2 \mathrm{eV}$ above $\epsilon_{F}$ at $100 \mathrm{GPa}$ The orbital is spread over skutterudite $\mathrm{N}_{4}$ rings, giving rise to electronic conductivity. 

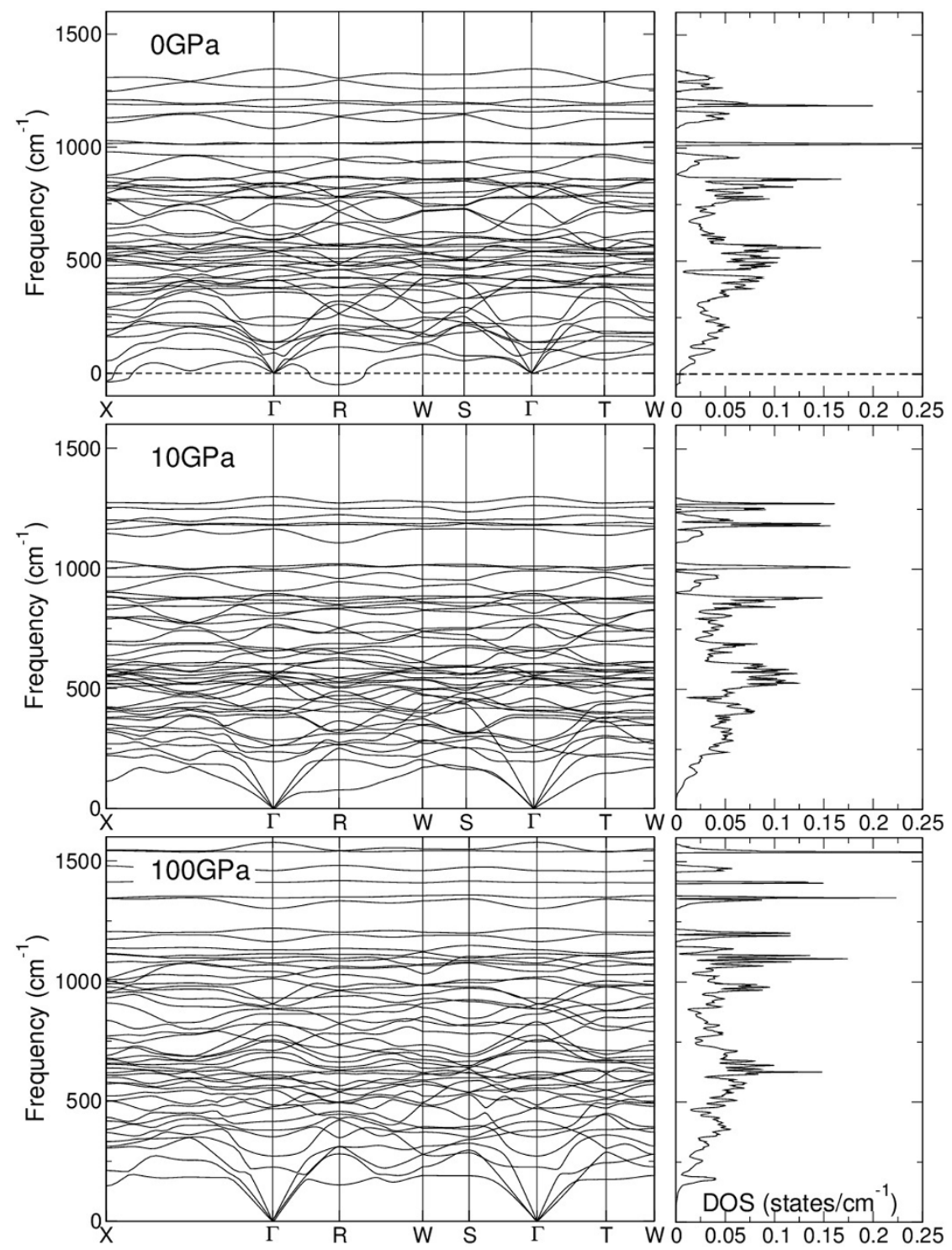

Figure 8 Phonon dispersion and phonon density of states for the Immm phase at $0 \mathrm{GPa}, 10 \mathrm{GPa}$ and $100 \mathrm{GPa}$.

pnictogen. It is characterised by an orthorhombic distortion, the extent of which increases as the pressure is decreased. Skutterudite$\mathrm{PN}_{3}$ is metallic throughout the range of pressures examined, 10$100 \mathrm{GPa}$, its electrical conductivity arising from the delocalisation of nitrogen p-states across $\mathrm{N}_{4}$ rings. Most significantly, it becomes a good electron-phonon superconductor with a $T_{c}$ of around $18 \mathrm{~K}$ at $10 \mathrm{GPa}$ due to strong electron-phonon coupling with low-frequency phonon modes. At higher pressures the superconducting $T_{c}$ is suppressed as low-energy acoustic modes gain energy. The superconducting behavior of $\mathrm{PN}_{3}$ is comparable to other high-pressure superconductors such as $\mathrm{Te}, \mathrm{Li}$ and $\mathrm{S}$, where the evolution of $T_{c}$ under

Table II | Calculated $\lambda, \omega_{\log }$ and $T_{c}$ values for the $/ m m m-\mathrm{PN}_{3}$ skutterudite. $T_{c}$ is calculated with two different values for the Coulomb pseudopotential $\mu^{*}$

\begin{tabular}{lcccc}
$\mathrm{P}(\mathrm{GPa})$ & $\lambda$ & $\omega_{\text {log }}(\mathrm{meV})$ & $T_{c}\left[\mu^{*}=0.10\right](\mathrm{K})$ & $T_{c}\left[\mu^{*}=0.13\right](\mathrm{K})$ \\
\hline 10 & 1.05 & 21.5 & 18.6 & 16.1 \\
20 & 0.77 & 31.4 & 15.5 & 12.4 \\
50 & 0.41 & 56.4 & 3.0 & 1.4 \\
100 & 0.39 & 59.9 & 2.3 & 0.9
\end{tabular}

pressure is determined by the behavior of low-energy phonon modes, and is enhanced close to dynamical instabilities ${ }^{40-42}$.

The phonon dispersion at $10 \mathrm{GPa}$ shows no sign of instability, opening the door to the possibility of its synthesis, perhaps by chemical synthesis from precursors in the correct ratio, or thin film deposition on a substrate with a similar lattice parameter. Furthermore, although the dodecahedral cage is too small to host one guest atom $(\mathrm{H}, \mathrm{Li}, \mathrm{Be})$ per primitive cell, it may be possible to stabilise the $\mathrm{PN}_{3}$ lattice at ambient pressure using off-stoichiometry doping with a guest species.

\section{Methods}

The USPEX code ${ }^{6,7}$ was used in conjunction with DFT to determine the most thermodynamically stable PN crystal structures for several different compositions.

USPEX employs evolutionary algorithms to find the atomic structure with the lowest enthalpy at a given pressure, given knowledge only of the chemical composition of the unit cell. It has been employed with great success with many other systems at high pressure $^{43-46}$.

All structures were optimised with the CASTEP DFT $\operatorname{code}^{47}$ at the GGA level, using the Perdew-Burke-Ernzerhof (PBE) exchange-correlation functional ${ }^{48}$. All lattice parameters and atomic positions were relaxed without constraints until forces varied by less than $0.05 \mathrm{eV}^{-1}$ and energies by less than $1 \times 10^{-5} \mathrm{eV}$ between BFGS steps. SCF steps were considered to be converged when the total energy varied by less than 1 $\times 10^{-6} \mathrm{eV}$ between steps. Electronic wavefunctions were expanded using a plane wave basis set with a cutoff of $350 \mathrm{eV}$, and core electrons $\left(1 \mathrm{~s}^{2}\right.$ in the case of $\mathrm{N}$ and 


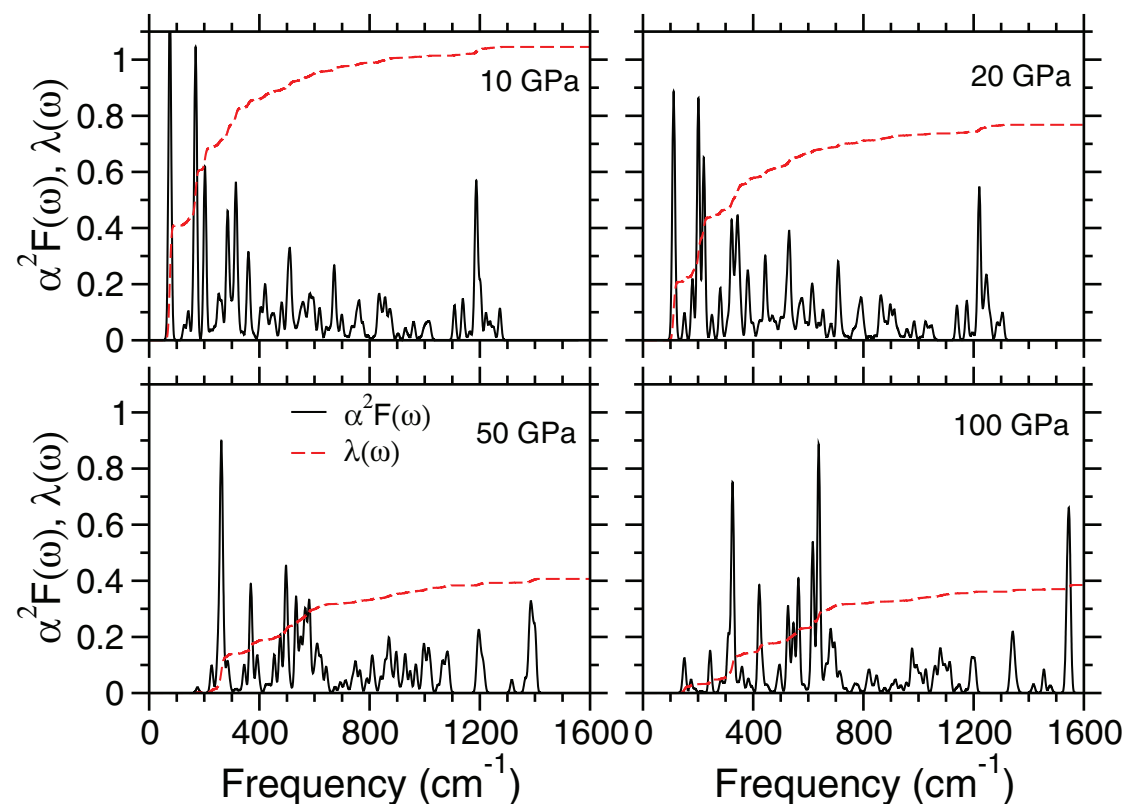
Figure $9 \mid$ Eliashberg function $\alpha^{2} F(\omega)$ and the integrated electron-phonon coupling constant $\lambda(\omega)=2 \int_{0}^{\omega} \mathrm{d} \omega^{\prime} \alpha^{2} F\left(\omega^{\prime}\right) / \omega^{\prime}$ for the Immm phase at
$10 \mathrm{GPa}, 20 \mathrm{GPa}, 50 \mathrm{GPa}$ and $100 \mathrm{GPa}$.

$1 \mathrm{~s}^{2} 2 \mathrm{~s}^{2} 2 \mathrm{p}^{6}$ in the case of $\mathrm{P}$ ) were represented with ultrasoft pseudopotentials. Monkhorst-Pack grids were generated per cell, using a spacing of $2 \pi \times 0.05 \AA^{-1}$. Ultrasoft pseudopotentials were employed with the intention of minimising calculation times; the nitrogen potential in particular had a core radius of $1.4 a_{0}$, which may have resulted in core overlap in the case of structures with extremely short $\mathrm{N}-\mathrm{N}$ bonds; however, enthalpies were compared with a hard reference pseudopotential with a $1.0 a_{0}$ core radius, and the results found to be consistent.

Phonon and electron-phonon calculations were performed on the 16-atom primitive cell of the $I \mathrm{mmm}$ skutterudite structure using density-functional perturbation theory (DFPT) $)^{49}$ as implemented in the QUANTUM ESPRESSO code ${ }^{50}$. Ultrasoft pseudopotentials, a plane wave cutoff of $60 \mathrm{Ry}$, a density cutoff of $600 \mathrm{Ry}$ and a $4 \times 4 \times 4$ Monkhorst-Pack grid for the electronic integrations were used throughout. The cell was fully reoptimised using the new pseudopotentials, and selected conduction bands were projected onto maximally localised Wannier functions (MLWF) using the wannier90 $\operatorname{code}^{51,52}$. Phonon frequencies and electron-phonon coefficients were calculated on a $2 \times 2 \times 2$ qpoint grid.

The Eliashberg functions of the electron-phonon coupling were calculated as

$$
\begin{aligned}
\alpha^{2} F(\omega) & =\frac{1}{N(0) N_{k} N_{q}} \sum_{\mathbf{k} \mathbf{q} n m \mu}\left|g_{\mathbf{k} n, \mathbf{k}+\mathbf{q} m}^{\mu}\right|^{2} \delta\left(\epsilon_{\mathbf{k} n}\right) \delta\left(\epsilon_{\mathbf{k}}+\mathbf{q} m\right) \\
& \times \delta\left(\omega-\omega_{\mu}(\mathbf{q})\right) .
\end{aligned}
$$

In Eq. (1) $g_{\mathbf{k} n, \mathbf{k}+\mathbf{q} m}^{\mu}=\sum_{s \alpha}\left\langle\mathbf{k} n\left|\delta V / \delta u^{s \alpha}(\mathbf{q})\right| \mathbf{k}+\mathbf{q} m\right\rangle \epsilon_{\mu}^{s \alpha}(\mathbf{q}) / \sqrt{2 M_{s} \omega_{\mu}(\mathbf{q})}$ is the electron-phonon matrix element, where $|\mathbf{k} n\rangle$ is a Kohn-Sham state with energy $\epsilon_{\mathbf{k} n}$ measured from the Fermi level $\left(\epsilon_{F}\right), N_{k}$ and $N_{q}$ are the number of electron and phonon momentum points used for the Brillouin zone sampling, $N(0)$ is the density of states per spin at $\epsilon_{F}, V$ is the self-consistent Kohn-Sham potential, $u^{s \alpha}(\mathbf{q})$ is the Fourier transformed displacement from equilibrium of atom $s$ along Cartesian direction $\alpha, M_{s}$ is the mass of atom $s$, and $\omega_{\mu}(\mathbf{q})$ and $\epsilon_{\mu}^{s \alpha}(\mathbf{q})$ are respectively phonon frequency and polarisations with momentum $\mathrm{q}$. A finer $12 \times 12 \times 12$ mesh was used in the sum over k-points in Eq. (1). Superconducting $T_{c}$ values were estimated from the Allen-Dynes modified McMillan equation ${ }^{53}$ :

$$
T_{c}=\frac{\omega_{\log }}{1.2} \exp \left(-\frac{1.04(1+\lambda)}{\lambda-\mu *(1-0.62 \lambda)}\right),
$$

where the electron-phonon coupling constant is calculated as

$$
\lambda=2 \int_{0}^{\infty} \mathrm{d} \omega \frac{\alpha^{2} F(\omega)}{\omega}
$$

the logarithmic frequency average is $\omega_{\log }=\exp \left(\frac{2}{\lambda} \int_{0}^{\infty} \mathrm{d} \omega \frac{\alpha^{2} F(\omega)}{\omega} \ln \omega\right)$, and $\mu^{*}$ is the Coulomb pseudopotential parameter.

1. McMillan, P. F. New materials from high-pressure experiments. Nature Mater $\mathbf{1}$, 19 (2002).
2. Solozhenko, V. L. \& Gregoryanz, E. Synthesis of superhard materials. Mater. Today 8, 44 (2005)

3. McMillan, P. F. High-pressure synthesis of materials. In High-Pressure Crystallography: from fundamental phenomena to technological applications, 373 (Springer, 2010).

4. Zerr, A., Miehe, G. \& Riedel, R. Synthesis of cubic zirconium and hafnium nitride having $\mathrm{Th}_{3} \mathrm{P}_{4}$ structure. Nature Mater. 2, 185 (2003).

5. Zhang, W. et al. Unexpected stable stoichiometries of sodium chlorides. Science 342, 1502 (2013)

6. Oganov, A. R. \& Glass, C. W. Crystal structure prediction using ab initio evolutionary techniques: principles and applications. J. Chem. Phys. 124, 244704 (2006).

7. Oganov, A. R. \& Glass, C. W. Evolutionary crystal structure prediction as a tool in materials design. J. Phys.: Condens. Matter 20, 064210 (2008).

8. Pickard, C. J. \& Needs, R. J. Ab initio random structure searching. J. Phys.: Condens. Matter 23, 053201 (2011).

9. Wang, Y., Lv, J., Zhu, L. \& Ma, Y. Crystal structure prediction via particle-swarm optimization. Phys. Rev. B 82, 094116 (2010).

10. Buzea, C. \& Robbie, K. Assembling the puzzle of superconducting elements: a review. Supercond. Sci. Technol. 18, R1 (2005).

11. Xie, Y., Oganov, A. R. \& Ma, Y. Novel high pressure structures and superconductivity of $\mathrm{CaLi}_{2}$. Phys. Rev. Lett. 104, 177005 (2010).

12. Martinez-Canales, M. et al. Novel structures and superconductivity of silane under pressure. Phys. Rev. Lett. 102, 087005 (2009).

13. Gao, G. et al. Dissociation of methane under high pressure. Proc. Natl. Acad. Sci. USA 107, 1317 (2010).

14. Zhu, L. et al. Substitutional alloy of bi and te at high pressure. Phys. Rev. Lett. 106, 145501 (2011).

15. Errea, I., Martinez-Canales, M. \& Bergara, A. Ab initio study of superconducting hexagonal $\mathrm{Be}_{2} \mathrm{Li}$ under pressure. Phys. Rev. B 78, 172501 (2008).

16. Raza, Z., Pickard, C. J., Pinilla, C. \& Saitta, A. M. High energy density mixed polymeric phase from carbon monoxide and nitrogen. Phys. Rev. Lett. 111, 235501 (2013).

17. Matthias, B. T. \& Hulm, J. K. A search for new superconducting compounds. Phys. Rev. 87, 799 (1952).

18. Yamanaka, S., Hotehama, K. \& Kawaji, H. Superconductivity at 25.5 k in electrondoped layered hafnium nitride. Nature Lett. 392, 580 (1998).

19. Wang, C. et al. First-principles calculations on the mechanical properties of niobium nitrides. Solid State Commun. 149, 725 (2009).

20. Taguchi, Y., Kitora, A. \& Iwasa, Y. Increase in $t_{c}$ upon reduction of doping in $\mathrm{Li}_{\mathrm{x}} \mathrm{ZrNCl}$ superconductors. Phys. Rev. Lett. 97, 107001 (2006).

21. Sofo, J. O. \& Mahan, G. D. Electronic structure of $\mathrm{CoSb}_{3}$ : a narrow-band-gap semiconductor. Phys. Rev. B 58, 620 (1998).

22. Möchel, A. et al. Lattice dynamics in the $\mathrm{FeSb}_{3}$ skutterudite. Phys. Rev. B $8 \mathbf{4}$ 064302 (2011).

23. Meisner, G. P. Superconductivity and magnetic order in ternary rare earth transition metal phosphides. Physica B + C 108, 763 (1981).

24. Slack, G. A. \& Tsoukala, V. G. Some properties of semiconducting $\mathrm{IrSb}_{3}$. J. Appl. Phys. 76, 1665 (1994). 
25. Jung, D., Whangbo, M. \& Alvarex, S. Importance of the $\mathrm{X}_{4}$ ring orbitals for the semiconducting, metallic, or superconducting properties of skutterudites $\mathrm{MX}_{3}$ and $\mathrm{RM}_{4} \mathrm{X}_{12}$. Inorg. Chem. 29, 2252 (1990).

26. Shirotani, I. et al. Superconductivity of filled skutterudites $\mathrm{LaRu}_{4} \mathrm{As}_{12}$ and $\mathrm{PrRu}_{4} \mathrm{As}_{12}$. Phys. Rev. B 56, 7866 (1997).

27. Shirotani, I. et al. Superconductivity of the new skutterudite compound $\mathrm{La}_{x} \mathrm{Rh}_{4} \mathrm{P}_{1} 2$ at high pressure. J. Phys.: Condens. Matter 17, 7353 (2005).

28. Bauer, E. et al. Superconductivity in novel ge-based skutterudites: $\{\mathrm{Sr}, \mathrm{Ba}\} \mathrm{Pt}_{4} \mathrm{Ge}_{12}$. Phys. Rev. Lett. 99, 217001 (2007).

29. Bauer, E. et al. Superconductivity and spin fluctuations in $\{\mathrm{Th}, \mathrm{U}\} \mathrm{Pt}_{4} \mathrm{Ge}_{12}$ skutterudites. Phys. Rev. B 78, 064516 (2008).

30. Bettermann, G., Krause, W., Riess, G. \& Hofmann, T. Phosphorus compounds, inorganic. Ullman's Encyclopedia of Industrial Chemistry 27, 1 (2012).

31. Schnick, W., Lu, J. \& Krumeich, F. Phosphorus nitride $\mathrm{P}_{3} \mathrm{~N}_{5}$ : synthesis, spectroscopic and electron microscopic investigations. Chem. Mater. 8, 281 (1996).

32. Horstmann, S., Irran, E. \& Schnick, W. Synthesis and crystal structure of phosphorus (v) nitride $\alpha-\mathrm{P}_{3} \mathrm{~N}_{5}$. Angew. Chem. Int. Ed. 36, 1873 (1997)

33. Landskron, K., Huppertz, H., Senker, J. \& Schnick, W. High-pressure sysnthisis of $\gamma-\mathrm{P}_{3} \mathrm{~N}_{5}$ at $11 \mathrm{gpa}$ and $1500^{\circ} \mathrm{c}$ in a multianvil assembly: a binary phosphorus (v) nitride with a three-dimensional network structure from $\mathrm{PN}_{4}$ tetrahedra and tetragonal $\mathrm{PN}_{5}$ pyramids. Angew. Chem. Int. Ed. 40, 2643 (2001).

34. Dong, J., Kinkhabwala, A. A. \& McMillan, P. F. High-pressure polymorphism in phosphorus nitrides. Phys. Status Solidi (B) 241, 2319 (2004).

35. Kroll, P. \& Schnick, W. A density functional study of phosphorus nitride $\mathrm{P}_{3} \mathrm{~N}_{5}$ : refined geometries, properties and relative stability of $\alpha-\mathrm{P}_{3} \mathrm{~N}_{5}$ and $\gamma-\mathrm{P}_{3} \mathrm{~N}_{5}$ and a further possible high-pressure phase $\delta-\mathrm{P}_{3} \mathrm{~N}_{5}$ with kyanite-type structure. Chem. Eur. J. 8, 3530 (2002).

36. Pellicer-Porres, J., Saitta, A. M., Polian, A., Itié, J. P. \& Hanfland, M. Six-foldcoordinated phosphorus by oxygen in $\mathrm{AlPO}_{4}$ quartz homotype under pressure. Nature Mater. 6, 698 (2007).

37. Llunell, M., Alemany, P., Alvarez, S., Vernes, A. \& Zhukov, V. P. Electronic structure and bonding in skutterudite-type phosphides. Phys. Rev. B 53, 10605 (1996).

38. Vining, C. B. Half-full glasses: the new black. Nature Mater. 7, 765 (2008).

39. Mihaly, L. Crystal cages for clean coolers. Nature 395, 839 (1998).

40. Mauri, F., Zakharov, O., de Gironcoli, S., Louie, S. G. \& Cohen, M. L. Atomic structure of icosahedral $\mathrm{B}_{4} \mathrm{C}$ boron carbide from a first principles analysis of $\mathrm{nmr}$ spectra. Phys. Rev. Lett. 77, 1151 (1996).

41. Profeta, G. et al. Superconductivity in lithium, potassium, and aluminium under extreme pressure: a first-principles study. Phys. Rev. Lett. 96, 047003 (2006).

42. Degtyareva, O. et al. Competition of charge-density waves and superconductivity in sulfur. Phys. Rev. Lett. 99, 155505 (2007).

43. Oganov, A. R. et al. Ionic high-pressure form of elemental boron. Nature 457, 863 (2009).

44. Ma, Y. et al. Transparent dense sodium. Nature 458, 182 (2009).

45. Oganov, A. R. et al. Exotic behavior and crystal structures of calcium under pressure. Proc. Natl. Acad. Sci. USA 107, 7646 (2010).

46. Oganov, A. R., Glass, C. W. \& Ono, S. High-pressure phases of $\mathrm{CaCO}_{3}$ : crystal structure prediction and experiment. Earth Planet Sci. Lett. 241, 95 (2006).
47. Clark, S. J. et al. First principles methods using castep. Z. Kristall. 220, 567 (2005)

48. Perdew, J. P., Burke, K. \& Ernzerhof, M. Generalized gradient approximation made simple. Phys. Rev. Lett. 77, 3865 (1996).

49. Baroni, S., de Gironcoli, S., Dal Corso, A. \& Giannozzi, P. Phonons and related crystal properties from density-functional perturbation theory. Rev. Mod. Phys. 73, 515 (2001).

50. Giannozzi, P. et al. QUANTUM ESPRESSO: a modular and open-source software project for quantum simulations of materials. J. Phys.: Condens. Matter 21, 395502 (2009).

51. Mostofi, A. A. et al. wannier90: A tool for obtaining maximally-localised wannier functions. Comp. Phys. Comm. 178, 685 (2008).

52. Souza, I., Marzari, N. \& Vanderbilt, D. Maximally localized wannier functions for entangled energy bands. Phys. Rev. B 65, 035109 (2001).

53. Allen, P. B. \& Dynes, R. C. Transition temperature of strong-coupled superconductors reanalyzed. Phys. Rev. B 12, 905 (1975).

\section{Acknowledgments}

Z.R. and A.M.S. acknowledge the GENCI IDRIS French National supercomputing facility for CPU time (Project Grant No. 91387), and thank the French Agence Nationale de la Recherche (ANR) for support through Project No. ANR-2011-BS08-018. I.E. would like to acknowledge financial support from the Department of Education, Language Policy and Culture of the Basque Government (Grant No. BFI-2011-65). A.R.O. thanks the National Science Foundation (EAR-1114313, DMR-1231586), DARPA (Grants No. W31P4Q1210008 and W31P4Q1310005), the Government (No. 14.A12.31.003) and the Ministry of Education and Science of the Russian Federation (Project No. 8512) for financial support, and Foreign Talents Introduction and Academic Exchange Program (No. B08040).

\section{Author contributions}

Z.R. and I.E. performed the calculations, A.R.O. and A.M.S. contributed to the analysis. Z.R. and I.E. wrote the manuscript.

\section{Additional information}

Competing financial interests: The authors declare no competing financial interests.

How to cite this article: Raza, Z., Errea, I., Oganov, A.R. \& Saitta, A.M. Nove superconducting skutterudite-type phosphorus nitride at high pressure from first-principles calculations. Sci. Rep. 4, 5889; DOI:10.1038/srep05889 (2014).

This work is licensed under a Creative Commons Attribution-NonCommercialShareAlike 4.0 International License. The images or other third party material in this article are included in the article's Creative Commons license, unless indicated otherwise in the credit line; if the material is not included under the Creative Commons license, users will need to obtain permission from the license holder in order to reproduce the material. To view a copy of this license, visit http:// creativecommons.org/licenses/by-nc-sa/4.0/ 